this. First, the well-known methodological difficulties in cross-national studies of elderly suicides (Shah et al., 2007) may be important. Second, life-long adversity has components other than economic adversity and includes education, housing, employment, status in society and access to healthcare and social welfare. These variables were not measured in this study. Third, measures of economic adversity were only available for a relatively recent period and it is possible that exposure to economic adversity early in life may be more important. Finally, the findings may be genuine.

\title{
References
}

Lindesay, J. (1991). Suicide in the elderly. International fournal of Geriatric Psychiatry, 6, 355-361.

McIntosh, J. L. (1984). Components of the decline in elderly suicides: suicide in young old and old old by race and sex. Death Education, 8, 113-124.

Seiden, R. H. (1981). Mellowing with age: factors affecting the non-white suicide rate. International fournal of Aging and Human Development, 13, 265-284.

Shah, A. K., Bhat, R., MacKenzie, S. and Koen, C. (2007). A cross-national study of the relationship between elderly suicide rates and life expectancy and markers of socio-economic status and healthcare. International Psychogeriatrics. E-published ahead of print publication.
AJIT SHAH
Institute of Philosophy, Diversity and Mental Health, Centre for Ethnicity and Health, University of Central Lancashire, Preston, U.K.
Professor of Ageing, Ethnicity and Mental Health, University of Central Lancashire, Preston, United Kingdom and Consultant Psychiatrist, West London Mental Health NHS Trust, London, United Kingdom Email: ajit.shah@wlmht.nhs.uk

\section{Depot risperidone in elderly patients: the experience of an Australian aged psychiatry service}

Antipsychotic medications form the mainstay of both the acute and maintenance treatment of schizophrenia. In recent years, atypical antipsychotics like risperidone, olanzapine and clozapine have come to be preferred because of their lower incidence of extra-pyramidal, anti-cholinergic and cardiac side-effects and a possible greater efficacy in reducing negative and neuro-cognitive symptoms (Ritchie et al., 2006).

Age-related pharmacokinetic changes, and higher rates of physical comorbidity and polypharmacy, make older people especially vulnerable to the adverse effects of medications. Atypical antipsychotics, with their "cleaner" side-effect 
profile, have naturally proved popular in aged psychiatry circles (Greve and O'Connor, 2005), but not all patients agree to take tablets.

The advent of depot risperidone, the first atypical preparation available in slow-release injectable format, offered an attractive treatment alternative for this vulnerable patient group. One of our aged mental health services was fortunate to get funding for depot risperidone before it became freely available in Australia and this is a brief account of our experience with it. Note that informed consent was obtained from those patients who might be identifiable in this published version of our work.

We conducted a retrospective file review of all the aged patients in the Dandenong area who were treated with depot risperidone from October 2003 to December 2006. No rating scales were used at that time on a regular basis. The 18 patients (14 females and four males), who ranged in age from 66 to 84 years (mean 70.5 years), had taken depot risperidone for an average of 17.2 months (range three to 38 months). Seventeen had a diagnosis of schizophrenia or schizoaffective disorder and one had Alzheimer's disease with prominent psychotic symptoms. Their duration of illness ranged from three to 51 years (mean 35 years). Fourteen of them had previously been prescribed typical antipsychotic depot injections because of a refusal to take tablets.

We initiated 12 patients on depot risperidone as inpatients, four as outpatients and two were transferred to our service on it for ongoing care. All were started on a $25 \mathrm{mg}$ fortnightly dose. Fifteen were cross-titrated from oral risperidone 1 to $2 \mathrm{mg}$ daily over a two to four week period: two were switched directly to depot risperidone because of a refusal to comply with oral treatment for even a short period of time, and one was switched directly from another oral atypical. Twelve patients were subsequently managed on a fortnightly dose of $37.5 \mathrm{mg}$, three on $50 \mathrm{mg}$, and one each on $25 \mathrm{mg}$ and $75 \mathrm{mg}$. One was managed on a four-weekly regime of $37.5 \mathrm{mg}$. It appeared that $25 \mathrm{mg}$ per fortnight was too low a dose for most patients.

Overall, our experience with depot risperidone in elderly patients was similar to that of Keks et al. (2003) in a younger population. All patients experienced some symptomatic improvement and four became free of all positive and negative symptoms. Three of them accepted treatment voluntarily from the outset and four of the 15 patients who were subject initially to involuntary treatment orders came to be managed on a voluntary basis. Two of the four chose to remain on injections and one agreed to take a $1 \mathrm{mg}$ oral dose. The fourth developed prominent extra-pyramidal side effects (EPSE) on $37.5 \mathrm{mg}$ and was switched successfully to an alternative oral agent.

As a group, elderly females are most prone to EPSE (Jeste et al., 1996). It was of interest, therefore, to note its emergence in our small but predominantly female sample. One male and six female patients had a history of EPSE on 
other treatments. All showed improvement on depot risperidone and two women were symptom-free. A further two women, with no past EPSE, developed Parkinsonism and akathisia on doses of $37.5 \mathrm{mg}$ and $25 \mathrm{mg}$ respectively. The former improved significantly on a different oral antipsychotic: the other improved partially on a combination of $25 \mathrm{mg}$ injections and an anti-cholinergic medication. She would not comply with oral medication.

One patient complained of sedation on $37.5 \mathrm{mg}$ and another had modest weight gain on the same dose but neither requested or needed a change in treatment. We were concerned that 17 of the 18 patients had one or more cardiovascular risk factors given evidence that atypicals might be responsible for small increases in "cerebrovascular events" and mortality in people with dementia (Committee on Safety of Medications, 2004). One of our patients had had a transient ischemic attack while on depot risperidone but recovered fully and remains well. There were no other new vascular events. The patient with Alzheimer's disease had no side-effects at all. His mental state improved to a point where we ceased injections and treated him instead with $1 \mathrm{mg}$ oral risperidone and a cognitive enhancer.

It is recommended by its manufacturer that depot risperidone be given at two-weekly intervals (Janssen-Cilag, 2003). In general we agree with this. Attempts to reduce the frequency, or give a higher dose at longer intervals, proved unsuccessful in two cases. In one instance, we reverted to a fortnightly regime of $37.5 \mathrm{mg}$ with subsequent improvement. With the second patient, who is still not symptom-free, we persisted with a four-weekly regime of $37.5 \mathrm{mg}$ because the patient found the injections so unpleasant. Readmission to hospital has not been required.

Overall, our initial experience with depot risperidone was promising. It was generally well tolerated with good improvement of both positive and negative symptoms. It has become an important part of our treatment armoury for patients with chronic psychoses who require a depot medication.

\section{Conflict of interest}

None.

\section{References}

Committee on Safety of Medicines (2004). Atypical anti-psychotic drugs and stroke. http://www.mca.gov.uk/au work/monitorsafequalmed/safetymessages/ atypicalantipsychotic_qa.htm. Last accessed 23 May 2007.

Greve, M. and O'Connor, D. (2005). A survey of Australian and New Zealand old age psychiatrists' preferred medications to treat behavioural and psychological symptoms of dementia. International Psychogeriatrics, 17, 165-178. 
Janssen-Cilag (2003). Risperdal Consta intra-muscular injection: product information. Sydney: Janssen-Cilag Pty Ltd.

Jeste, D. V. et al. (1996). Risk of tardive dyskinesia in older patients: a prospective longitudinal study of 266 outpatients. Archives of General Psychiatry, 57, 756-765.

Keks, N. et al. (2003). Initial experience with long acting Risperidone microspheres injection. Australasian Psychiatry, 11, 273-275.

Ritchie, C. W. et al. (2006). A comparison of the efficacy and safety of olanzapine and risperidone in the treatment of elderly patients with schizophrenia: an open study of six months duration. International fournal of Geriatric Psychiatry, 21, 171-179.

\section{DHIREN SINGH AND DANIEL W. O'CONNOR}

Department of Psychological Medicine, Monash University, and Southern Health, Melbourne, Australia

Email: dhiren.singh@southernhealth.org.au 